\title{
Vaccine Prevention of Acute Otitis Media
}

David P. Greenberg, MD and Alejandro Hoberman, MD

\begin{abstract}
Address
Department of Pediatrics, University of Pittsburgh School of Medicine, Division of Allergy, Immunology and Infectious Diseases, Children's Hospital of Pittsburgh, Room 4B-320, 3705 Fifth Avenue, Pittsburgh, PA 152/3-2583, USA.

E-mail: greenbd@chplink.chp.edu
\end{abstract}

Current Allergy and Asthma Reports 200I, I:358-363

Current Science Inc. ISSN I529-7322

Copyright $(92001$ by Current Science Inc.

The incidence of acute otitis media (AOM) in infants and young children has increased dramatically in recent years in the United States. AOM often follows upper respiratory tract infections due to pathogens such as respiratory syncytial virus (RSV), influenza virus, and parainfluenza virus (PIV). These viruses cause eustachian tube dysfunction that is critical to the pathogenesis of AOM. Vaccines against these viruses would likely reduce the incidence of AOM. In three previous studies, influenza virus vaccines reduced the incidence of AOM by $30 \%$ to $36 \%$. Vaccines to prevent infections with RSV and PIV type 3 are undergoing clinical testing at this time. Streptococcus pneumoniae, nontypeable Haemophilus influenzae (NTHi), and Moraxella catarrhalis are the three most common AOM pathogens. Heptavalent pneumococcal conjugate vaccine is effective in preventing invasive disease and AOM caused by serotypes contained in the vaccine. Vaccine candidates for NTHi and $M$. catarrhalis are under development.

\section{Introduction}

For infants and young children, acute otitis media (AOM) is the most frequent diagnosis in the office setting [1]. In a study conducted by the Centers for Disease Control and Prevention, the annual number of office visits for AOM in the United States increased from 9.9 million in 1975 to 24.5 million in 1990 [2]. In addition, the rate of tympanostomy tube placement increased approximately fourfold between 1970 and 1995 [3]. In 1995, it was estimated that the direct and indirect cost of treating otitis media in children under 5 years of age in the United States was 5.0 billion dollars [4].

In a study of 2253 infants, Paradise et al. [5] found that in the 1 st and 2nd years of life, antibiotics for AOM were given for a mean of 42 and 49 days, middle ear effusion (MEE) was present during $20 \%$ and $17 \%$ of the time, and tympanostomy tubes were inserted in $1.8 \%$ and $4.2 \%$ of the infants, respectively. By 2 years of age, $91 \%$ of children experienced at least one episode of MEE. Children who develop their first episode of AOM before 6 months of age are more likely to develop recurrent disease [6]. In addition to young age, risk factors often associated with frequent episodes of AOM include male gender, race (eg, Native Americans), genetic predisposition (eg, siblings with frequent $\mathrm{AOM}$ ), underlying medical conditions (eg, cleft palate), low socioeconomic status, daycare attendance, exposure to cigarette smoke in the household, and lack of breast feeding $[5,6]$.

\section{Epidemiology and Pathogenesis of Acute Otitis Media}

The eustachian tube (ET) protects the middle ear against invading organisms by its mucociliary system and secreted antimicrobial substances such as defensins, lactoferrin, lysozyme, and surfactant [7]. In addition, serum antibodies transudate into the middle ear cavity, protecting it against specific bacterial pathogens [8]. These defenses may be disrupted by respiratory viruses, leading to impairment of ET function. In a chinchilla model, influenza A caused epithelial damage and accumulation of cellular and mucus debris in the lumen of the ET and subsequent negative middle ear pressure [9]. Similar evidence of ET dysfunction has been substantiated in studies of children. Abnormal tympanograms occurred in 75\% of children with clinical symptoms of an upper respiratory infection (URI), and $97 \%$ of the abnormalities were detected by the 4 th day of illness [10].

It appears that respiratory viruses affect ET function by inducing the release of various cytokines and other inflammatory mediators by epithelial cells [11]. In addition to ET dysfunction, some respiratory viruses have an immunosuppressive effect on polymorphonuclear cells and increase the adherence of bacteria to epithelial cells. Adenovirus increased binding of Streptococcus pneumoniae, and influenza A virus induced increased binding of S. pneumoniae and Haemophilus influenzae to human epithelial cells.

Numerous studies have confirmed the strong relationship between viral URI and AOM. Viral URI and AOM share many of the same risk factors including seasonality, young age, and daycare attendance $[5,6]$. AOM has a peak onset 3 to 4 days after the onset of a viral URI [12]. Despite the close association, previous studies have generally detected viruses in only approximately $10 \%$ of middle ear fluid specimens from children with AOM [13].

In a more recent study, Heikkinen et al. [14•] evaluated the prevalence of respiratory viruses in the middle ear fluid 
of 456 children aged 2 months to 7 years. Among children who had respiratory syncytial virus (RSV) detected in nasal wash specimens, 48 of 65 (74\%) had the same virus in their middle ear fluid. The corresponding proportion for children with parainfluenza virus (PIV) was 15 of 29 (52\%); influenza virus, 10 of 24 (42\%); enterovirus, three of $27(11 \%)$; and adenovirus one of $23(4 \%)$. Among middle ear samples from which viruses were isolated, viruses and bacteria were found together in 43 (65\%) of 66 ears, whereas viruses were the sole middle-ear pathogen in $22(35 \%)$ of 62 ears.

Using reverse transcriptase-polymerase-chain reaction (RT-PCR), Pitkaranta et al. [15•] detected viral RNA in $62 \%$ of nasal aspirates and $48 \%$ of MEEs during episodes of AOM. In children with positive nasal aspirate cultures, rhinovirus RNA was found in $64 \%$ of middle-ear fluid specimens, RSV in $57 \%$, and coronavirus in $36 \%$. Vaccines that prevent these common respiratory illnesses also will likely prevent AOM.

\section{Vaccine Prevention of Viral Respiratory Infections Associated with Acute Otitis Media} Acute otitis media is commonly associated with influenza infection, yet the licensed vaccine is not widely administered to healthy infants and young children. The efficacy of influenza vaccine in preventing AOM has been evaluated in three previous studies. In the first study, Heikkinen et al. [16] administered trivalent influenza vaccine to 187 children aged 1 to 4 years in 11 daycare centers and gave no vaccine to another 187 children in eight different daycare centers in Finland. During the influenza season, children were seen in the clinic for fever or signs of URI. The investigators were not blinded, but "in most cases" they did not know if the child had been vaccinated. Influenza occurred in five $(2.7 \%)$ vaccinees and $29(15.5 \%)$ control subjects. The efficacy of the vaccine against influenza and influenzaassociated AOM was $83 \%$ for each. AOM was diagnosed in $35(18.7 \%)$ vaccinees and $55(29.4 \%)$ control subjects, representing a $36 \%$ reduction in all episodes of AOM due to influenza vaccine.

In the second study, Clements et al. [17] evaluated 186 children aged 6 to 30 months who were enrolled in eight daycare centers in North Carolina. Some of the participants were enrolled in 1992-1993 and were randomly assigned to trivalent influenza vaccine or hepatitis $B$ vaccine. These children were given the same vaccine in 1993-1994, and new children were enrolled to receive influenza vaccine, ear examination only (by parental request), or ear examination only (by randomization). The children were examined biweekly by blinded investigators during the respiratory season. During the peak influenza season, $\mathrm{AOM}$ was diagnosed in $21.5 \%$ of children given influenza vaccine and $36.4 \%$ of children given hepatitis $B$ vaccine or no vaccine. The unadjusted reduction of incidence of $A O M$ was $32 \%(P=0.02)$ and of MEE was $25 \%(P=0.04)$.
In a third multicenter, double-blind, randomized study, a live cold-adapted trivalent influenza vaccine or placebo (2:1 ratio) was administered intranasally to 1602 children aged 15 to 71 months [ $18 \bullet \bullet$ ]. The children were seen by an investigator when symptoms consistent with influenza disease occurred. AOM was not a primary endpoint of the trial; thus no case definition was used. Fourteen of 1070 (1.3\%) vaccinees and 95 of 532 (17.9\%) placebo recipients developed influenza, for an efficacy rate of $93 \%$ (95\% CI, $88 \%$ to $96 \%$ ). The influenza vaccine reduced the incidence of febrile AOM by $30 \%(95 \% \mathrm{CI}$, $18 \%$ to $45 \%$ ).

Each of the above studies has one or more methodologic limitations, including 1) inadequate sample size, 2) lack of randomization, 3 ) inadequate blinding, 4) passive surveillance for influenza and AOM, and/or 5) lack of standard criteria for the definition of AOM. Despite these methodologic flaws, influenza vaccine was shown to reduce the incidence of AOM by a similar proportion in each study ( $30 \%$ to $36 \%$ ).

To address the limitations of the above studies, we have been conducting a double-blind efficacy trial of approximately 800 children aged 6 to 24 months who were randomly assigned (2:1 ratio) to receive inactivated trivalent influenza vaccine (Fluzone; Aventis Pasteur, Swiftwater, PA) or placebo during a 2-year period [19]. The first cohort of 417 children was enrolled in the fall of 1999 and seen biweekly through March 2000 then monthly from April through October 2000. The second cohort of 378 children was enrolled in the fall of 2000 and seen biweekly through March 2001.

In addition to scheduled visits, parents were instructed to contact us with signs or symptoms of respiratory infection or AOM. Strict case definitions were used to diagnose AOM and MEE using pneumatic otoscopy, tympanometry, spectral gradient acoustic reflectometry, and videootoscopy with computer capture images of the tympanic membrane saved in an electronic database. To detect influenza disease, viral throat cultures were obtained with each febrile respiratory illness or AOM. Also, nasopharyngeal colonization with penicillin-resistant $S$. pneumoniae was monitored throughout the trial.

The data are still blinded as of this writing. However, in the 1st-year cohort, preliminary results show that at least one episode of AOM developed in 226 (58\%) of 387 children during the respiratory season (mean, 1.17 episodes/child) and in 247 (66\%) of 374 children during the 1-year follow-up period (mean, 1.68 episodes/child). Culture-proven influenza occurred in 37 children in the first cohort. S. pneumoniae was isolated from 157 nasopharyngeal cultures ( 99 children, 1 to 7 positive cultures/ child); 23 (15\%) were penicillin resistant (minimal inhibitory concentration $[\mathrm{MIC}] \geq 2.0 \mu \mathrm{g} / \mathrm{mL}$ ) and four $(2.5 \%)$ were cefotaxime resistant (MIC $\geq 2.0 \mu \mathrm{g} / \mathrm{mL}$ ).

In addition to those for influenza, vaccines for other respiratory viruses would likely prevent AOM in young 
children. The most common upper and lower respiratory virus in young children is RSV. A vaccine to prevent RSV will need to be effective in infants less than 6 months of age, when the disease is most severe. Development of such vaccines is problematic because 1 ) neonates have poor immunologic responses to viral surface glycoproteins, and these responses are further hampered by the presence of maternal antibody; 2) RSV has significant antigenic diversity; and 3) previous studies of a formalin-inactivated RSV vaccine led to enhanced disease in children later exposed to wild-type virus.

Evidence that antibody to RSV prevents AOM was observed in infants given RSV immune globulin, intravenous [20]. This immunoglobulin preparation significantly reduced the overall incidence of AOM and of RSV-associated AOM. Recently, vaccine candidates have been developed using cold-adapted $(c p)$, temperature-sensitive $(t s)$ strains. Some advantages of these intranasally administered live attenuated strains are that they mimic natural infection, induce cellular and humoral immunity, and replicate on mucosal surfaces even in the presence of maternal antibody. The development of these strains has been difficult because of over- or underattenuation and genetic instability.

Two candidate vaccines (cpts530/1009 and cpts248/955) were attenuated, genetically stable, and immunogenic in chimpanzees, highly attenuated in human adults, and immunogenic in seronegative children [21]. However, the strains were shed in high titers and transmitted to seronegative placebo recipients with a frequency of $20 \%$ to $25 \%$. Another candidate vaccine (cptsRSV 248/404) was safe and immunogenic in seronegative children greater than 6 months of age but caused nasal congestion in infants aged 4 to 12 weeks [22]. In another strategy for developing live RSV vaccines, infectious clones of RSV have been derived using CDNA [23]. Mutations can be introduced or specific genes deleted to produce an ideal strain with the proper balance of attenuation, immunogenicity, and genetic stability.

Subunit vaccines containing F or G glycoproteins of RSV or both are being developed for use in older children and adults. Currently, naïve infants are not candidates for these vaccines because of the experience in the 1960s when formalin-inactivated vaccine induced enhanced disease in vaccinees when they were later exposed to wild-type RSV. F glycoprotein displays less antigenic variation between strains and is more immunogenic than G glycoprotein.

Two forms of purified F glycoprotein (PFP-1 and PFP2) subunit vaccines have been evaluated in clinical trials. In a study of children 18 to 36 months of age who had previously been hospitalized with RSV infection, PFP-1 vaccine was safe, immunogenic, and, during a 6-month follow-up period, protected vaccinees against clinical RSV disease [24]. Other investigators have confirmed the safety and immunogenicity of PFP-1 vaccine in children, with significant increases of antibody to the F glycoprotein, although the vaccine did not always protect against subsequent RSV infection [25]. A similar subunit vaccine, PFP-2, has proven safe and immunogenic in children with bronchopulmonary dysplasia, children with cystic fibrosis, and an elderly population [26-28]. In children with bronchopulmonary dysplasia, PFP-2 vaccine protected against subsequent RSV infection [26]. In contrast to formalin-inactivated RSV vaccines, PFP-1 and PFP-2 vaccines have not induced enhanced disease in vaccinees infected with wild-type RSV. Expanded clinical trials of subunit vaccines are ongoing in children and adults.

Parainfluenza virus is another common cause of upper and lower respiratory disease in infants and young children, often complicated by AOM. As with RSV, to significantly reduce the incidence of disease PIV vaccine will have to be effective in infants less than 6 months of age. Formalininactivated vaccines are capable of inducing neutralizing antibody but are not protective in animal models. Subunit vaccines have been developed using hemagglutininneuraminidase and F glycoproteins. Such vaccines were protective in a rodent model and well tolerated in seropositive adults but have not been studied extensively in humans. Live attenuated cpts strains of PIV administered by intranasal spray were protective in animal models, but an early candidate vaccine (cp18) caused rhinorrhea and wheezing in some seronegative young children [29].

A further attenuated candidate PIV3 vaccine (cp45) was tested in seronegative infants greater than 6 months of age [30]. The vaccine infected $86 \%$ of the recipients, induced a serum hemagglutination inhibition (HAI) antibody response in $81 \%$, and was well tolerated. Using another strategy, a bovine PIV type 3 (BPIV3) vaccine administered by intranasal spray was protective in animal models and was immunogenic and well tolerated in seropositive and seronegative children as young as 2 months of age. In a study by Karron et al. [31], 92\% of seronegative children 6 to 60 months of age were successfully infected with BPIV3 vaccine virus, $92 \%$ demonstrated an antibody response by HAI to BPIV3, and $61 \%$ developed a response to human PIV3 (HPIV3). In infants less than 6 months of age, BPIV3 vaccine was infectious in $92 \%$ of recipients, and the vaccine induced HAI antibody responses to BPIV3 in $67 \%$ and to HPIV3 in $42 \%$ of recipients [32]. Immunogenic interference was noted in infants with maternal antibody to PIV3.

In a recent study, infants were randomly assigned to receive BPIV3 vaccine or placebo by intranasal spray at 2,4 , 6 , and 12 months of age [33]. The vaccine was well tolerated, and after the first three doses it induced a fourfold rise of HAI antibodies to BPIV3 and resulted in nasal shedding in approximately $80 \%$ of the vaccinees. Further refinements of PIV vaccines are needed before an effective vaccine can be licensed for use in infants.

\section{Vaccine Prevention of Bacterial Causes of Acute Otitis Media}

The most frequent bacterial pathogens associated with AOM are S. pneumoniae (25\% to 50\%), nontypeable H. influenzae 
(NTHi, $15 \%$ to $30 \%$ ), Moraxella catarrhalis (3\% to 20\%), Streptococcus pyogenes (2\% to 3\%), and Staphylococcus aureus $(2 \%$ to $3 \%)$. Vaccines are being developed against all of these pathogens, but currently only vaccines against $S$. pneumoniae are licensed and available for use in the United States.

The first vaccines licensed to prevent infections caused by S. pneumoniae-initially 14-valent, followed by 23 valent pneumococcal polysaccharide vaccines-cannot be used in children less than 24 months of age, who are at highest risk for invasive pneumococcal disease and AOM. Polysaccharide antigens are T-cell independent and do not reliably induce antibody responses in children less than 24 months of age. In contrast, conjugation of bacterial polysaccharides to protein antigens induces T-cell memory and enhances immunologic responses in infants. The prototype of these vaccines, $H$. influenzae type b (Hib) conjugate vaccine, has dramatically reduced the incidence of invasive Hib infections, including meningitis, in the United States.

Similar methods were used to manufacture pneumococcal conjugate vaccine (PCV)-7, which contains the seven most common serotypes that cause invasive disease in children less than 6 years of age in the United States. PCV-7 (Prevnar; Wyeth-Lederle, Philadelphia, PA) is licensed in the United States and recommended for universal use beginning at 2 months of age [34]. The seven serotypes contained in the vaccine represent those most often resistant to penicillin and other antibiotics [35]. PCV-7 has been shown to prevent nasopharyngeal acquisition of pneumococcal serotypes contained in the vaccine [36].

In a double-blind, randomized efficacy trial conducted in Northern California's Kaiser Permanente health system,

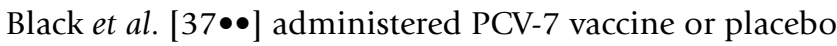
to 37,868 infants at $2,4,6$, and 12 to 15 months of age. In an intent-to-treat analysis, the efficacy of the vaccine in preventing invasive pneumococcal disease caused by serotypes covered in the vaccine was $93.9 \%$ (95\% CI, 79.6\% to $98.5 \%$ ). AOM was not a primary endpoint of the study, so no standardized case definition was used. In the intent-totreat analysis, the PCV-7 vaccine reduced the incidence of all episodes of AOM by $6.4 \%$ (95\% CI, 3.9\% to $8.7 \%$ ) and the use of tympanostomy tubes by $20.3 \%$ (95\% CI, $3.6 \%$ to $34.1 \%$ ).

In a separate study in Finland, 1662 infants were randomly assigned to receive PCV-7 vaccine or placebo at

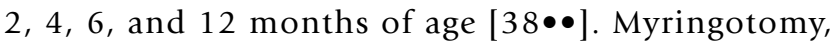
tympanocentesis, or both were performed during episodes of AOM. PCV-7 vaccine reduced the incidence of AOM from any cause by $6 \%$ (95\% CI, $-4 \%$ to $16 \%)$, from any pneumococcal serotype by $34 \%$ (95\% CI, $21 \%$ to $45 \%$ ), and from pneumococcal serotypes contained in the vaccine by $57 \%$ ( $95 \% \mathrm{CI}, 44 \%$ to $67 \%$ ). In contrast, episodes of AOM caused by pneumococcal serotypes not covered by the vaccine increased 33\%. Some investigators have found an increase of nasopharyngeal carriage by nonvaccine serotypes following immunization with PCVs [39].
Follow-up studies are required to learn whether non-PCV7 pneumococcal serotypes will replace current strains causing AOM and if these strains will develop resistance when exposed to antibiotics in children.

Pneumococcal conjugate vaccines with expanded coverage to nine and 11 serotypes are under development by several pharmaceutical companies and are being evaluated in a number of clinical trials. Although these vaccines will prevent slightly more episodes of AOM than PCV-7 vaccine, the potential for nonvaccine serotypes to become more prevalent still exists. Alternative vaccines, specifically those based on pneumococcal proteins, may offer an advantage of preventing all pneumococcal strains regardless of serotype.

Pneumococcal protein candidates include PspA, pneumolysin, PsaA, PspC, neuraminidase, and hyaluronidase [40]. In a rat model, PspA has been shown to protect against AOM [41]. Intranasal immunization with full-length native PspA or recombinant PsaA reduces nasal carriage of pneumococci in mice, but both proteins administered in combination have a greater effect [40]. Mucosal immunization with these proteins elicits circulating and mucosal antibody, and systemic antibody to PspA protects animals against sepsis. One significant advantage of using PspA as a vaccine candidate is that antibody to a single PspA is cross-reactive to heterologous PspAs, categorized into six clades using amino acid sequence data [42]. For some pneumococcal strains, synergistic protection against sepsis in mice has been observed when pneumolysin is added to PspA for immunization. Ultimately, it is likely that multiple pneumococcal proteins will be combined in human vaccines.

Vaccines against other bacterial pathogens causing middle-ear disease are under development, specifically against NTHi and $M$. catarrhalis. Cell-surface antigens of NTHi that may be used in vaccines include outer membrane proteins (OMPs), high molecular weight (HMW) proteins, pili, and fimbriae. Six major OMPs of NTHi have been labeled P1 through P6 in order of decreasing molecular weights [43]. Bactericidal activity of antibody to some of these proteins has been demonstrated, but their ability to protect against infection or enhance clearance of organisms in animal models has been variable. Some OMPs demonstrate significant heterogeneity between strains, limiting their usefulness as vaccine candidates. The greatest interest has focused on P6 because it is surface exposed, immunogenic, and highly conserved among strains of NTHi. P6 has been shown to induce bactericidal antibody that enhances pulmonary clearance of homologous and heterologous NTHi in animal models [44]. However, children prone to frequent episodes of AOM may not recognize P6 as an effective immunogen [45]. HMW proteins and pili are important for attachment of NTHi to respiratory epithelium. Immunization of chinchillas with HMW proteins and pili has provided some degree of protection against AOM. Passive antibody 
to P5 and protein D protected chinchillas against AOM and MEE due to NTHi [46].

Potential antigens for vaccine development against $M$. catarrhalis include OMPs, fimbriae, and lipooligosaccharide (LOS) [47]. OMPs and LOS are fairly well conserved across many strains. The chinchilla has been inadequate for evaluation of vaccine candidates to $M$. catarrhalis, and it has not been shown that antibodies to these antigens are protective against the development of AOM. Kyd et al. [48] have developed a murine model demonstrating enhanced pulmonary clearance of $M$. catarrhalis following mucosal immunization with killed organisms. Ubiquitous surface protein A elicits bactericidal antibodies in animals, immunized mice clear M. catarrhalis more quickly from the respiratory tract, adult humans have high titers of antibody to this protein, and clinical trials with this protein are in progress [49]. CD is a porin OMP that enhances pulmonary clearance of $M$. catarrhalis in mice, but no clinical studies have been conducted. Other surface antigens of M. catarrhalis are being evaluated as potential vaccine candidates, but there is much to be learned about their function and the activity of their respective antibodies.

\section{Conclusions}

Significant progress has been made in understanding the epidemiology and pathogenesis of AOM in infants and young children, but the need to prevent these infections is greater than ever before. The incidence and cost of treating AOM have increased significantly in recent years. Antibiotic resistance has become widespread in all regions of the United States. Use of effective vaccines is the most costefficient way to prevent AOM. Viral vaccines can be used to prevent URIs that commonly precede AOM. Influenza virus vaccine reduces the incidence of AOM by approximately one third during the influenza season, but the vaccine is not currently recommended for all children. Future vaccines for RSV and PIV should have even greater effects on reducing the incidence of AOM. Bacterial vaccines are also needed to prevent AOM. Recently licensed PCV will significantly reduce AOM caused by serotypes contained in the vaccine, but protein-based vaccines are needed to cover all strains of pneumococci. Vaccines to prevent AOM caused by NTHi and M. catarrhalis are under development.

\section{References and Recommended Reading} Papers of particular interest, published recently, have been highlighted as:

- Of importance

- Of major importance

1. Freid VM, Makuc DM, Rooks RN: Ambulatory health care visits by children: principal diagnosis and place of visit. Hyattsville: Centers for Disease Control and Prevention/National Center for Health Statistics; 1998. [Vital and Health Statistics 13:1-23.]

2. Schappert SM: Office visits for otitis media: United States, 1975-90. Hyattsville: Centers for Disease Control and Prevention/National Center for Health Statistics; 1992 [Vital and Health Statistics Adv Data 214:1-18.]
3. Daly KA, Hunter LL, Giebink GS: Chronic otitis media with effusion. Pediatr Rev 1999, 20:85-93.

4. Gates GA: Cost-effectiveness considerations in otitis media treatment. Otolaryngol Head Neck Surg 1996, 114:525-530.

5. Paradise JL, Rockette HE, Colborn $\mathrm{K}$, et al.: Otitis media in 2253 Pittsburgh-area infants: prevalence and risk factors during the first two years of life. Pediatrics 1997, 99:318-333.

6. Teele DW, Klein JO, Rosner B: Epidemiology of otitis media during the first seven years of life in children in greater Boston: a prospective, cohort study. J Infect Dis 1989, 160:83-94.

7. Lim DJ, Chun YM, Lee HY, et al.: Cell biology of tubotympanum in relation to pathogenesis of otitis media - a review. Vaccine 2001, 19:S17-S25.

8. Rynnel-Dagoo B, Agren K: The nasopharynx and the middle ear: inflammatory reactions in middle ear disease. Vaccine 2001, 19:S26-S31.

9. Giebink GS, Ripley ML, Wright PF: Eustachian tube histopathology during experimental influenza A virus infection in the chinchilla. Ann Otol Rhinol Laryngol 1987, 96:199-206.

10. Sanyal MA, Henderson FW, Stempel EC, et al.: Effect of upper respiratory tract infection on eustachian tube ventilatory function in the preschool child. J Pediatr 1980, 97:11-15.

11. Heikkinen T: Role of viruses in the pathogenesis of acute otitis media. Pediatr Infect Dis J 2000, 19:S17-S23.

12. Heikkinen T, Ruuskanen O: Temporal development of acute otitis media during upper respiratory tract infection. Pediatr Infect Dis J 1994, 13:659-661.

13. Ruuskanen $\mathrm{O}$, Arola M, Putto-Laurila A, et al:: Acute otitis media and respiratory virus infections. Pediatr Infect Dis $J$ 1989, 8:94-99.

14. Heikkinen T, Thint M, Chonmaitree T: Prevalence of various respiratory viruses in the middle ear during acute otitis media. N Engl J Med 1999, 340:260-264.

Using culture and antigen detection techniques, the investigators found RSV in the middle ear fluid of $74 \%$ of children in whom the same virus was found in nasopharyngeal secretions, PIV in 52\%, and influenza virus in $42 \%$.

15. Pitkaranta A, Virolainen A, Jero J, et al.: Detection of rhinovirus, respiratory syncytial virus, and coronavirus infections in acute otitis media by reverse transcriptase polymerase chain reaction. Pediatrics 1998, 102:291-295.

These investigators used RT-PCR to detect rhinovirus RNA in the middle ear fluid of $64 \%$ of children with the same virus in their nasopharyngeal secretions, RSV in 57\%, and coronavirus in 36\%.

16. Heikkinen $T$, Ruuskanen $\mathrm{O}$, Waris $\mathrm{M}$, et al.: Influenza vaccination in the prevention of acute otitis media in children. Am J Dis Child 1991, 145:445-448.

17. Clements DA, Langdon L, Bland C, Emmanuel W: Influenza A vaccine decreases incidence of otitis media in 6- to 30month-old children in day care. Arch Pediatr Adolesc Med 1995, 149:1113-1117.

18. $\bullet$ Belshe RB, Mendelman PM, Treanor I, et al.: The efficacy of live attenuated, cold-adapted, trivalent, intranasal influenza virus vaccine in children. $N$ Engl J Med 1998, 338:1405-1412.

A novel trivalent, live attenuated, intranasal influenza vaccine was $93 \%$ efficacious against culture-positive influenza and resulted in $30 \%$ fewer episodes of febrile AOM.

19. Hoberman A, Greenberg DP, Paradise JL, et al.: Efficacy of influenza vaccine in the prevention of acute otitis media (AOM) [abstract]. Pediatr Res 2001, in press.

20. Simoes EAF, Groothuis JR, Tristram DA, et al.: Respiratory syncytial virus-enriched globulin for the prevention of acute otitis media in high-risk children. J Pediatr 1996, 129:214-219.

21. Karron RA, Wright PF, Crowe JE Jr, et al.: Evaluation of two live, cold-passaged, temperature-sensitive respiratory syncytial virus vaccines in chimpanzees and in human adults, infants, and children. J Infect Dis 1997, 176:1428-1436.

22. Wright PF, Karron RA, Crowe JE Jr, et al.: Evaluation of a live, attenuated respiratory syncytial virus (RSV) vaccine candidate, cpts 248/404, in infancy. Pediatr Res 1998, 43:161A.

23. Collins PL, Whitehead SS, Bukreyev A, et al.: Rational design of live-attenuated recombinant vaccine virus for human respiratory syncytial virus by reverse genetics. Adv Virus Res 1999, 54:423-451. 
24. Tristram DA, Welliver RC, Mohar CK, et al.: Immunogenicity and safety of respiratory syncytial virus subunit vaccine in seropositive children 18-36 months old. J Infect Dis 1993, 167:191-195.

25. Belshe RB, Anderson EL, Walsh EE: Immunogenicity of purified $\mathrm{F}$ glycoprotein of respiratory syncytial virus: clinical and immune responses to subsequent natural infection in children. J Infect Dis 1993, 168:1024-1029.

26. Groothuis JR, King SJ, Hogerman DA, et al.: Safety and immunogenicity of a purified $F$ protein respiratory syncytial virus (PFP-2) vaccine in seropositive children with bronochopulmonary dysplasia. J Infect Dis 1998, 177:467-469.

27. Piedra PA, Grace S, Jewell A, et al.: Purified fusion protein vaccine protects against lower respiratory tract illness during respiratory syncytial virus season in children with cystic fibrosis. Pediatr Infect Dis J 1996, 15:23-31.

28. Falsey AR, Walsh EE: Safety and immunogenicity of a respiratory syncytial virus subunit vaccine (PFP-2) in the institutionalized elderly. Vaccine 1997, 15:1130-1132.

29. Belshe RB, Karron RA, Newman FK, et al.: Evaluation of a live attenuated, cold-adapted parainfluenza virus type 3 vaccine in children. J Clin Microbiol 1992, 30:2064-2070.

30. Karron RA, Wright PF, Newman FK, et al.: A live human parainfluenza type 3 virus vaccine is attenuated and immunogenic in healthy infants and children. J Infect Dis 1995, 172:1445-1450.

31. Karron RA, Wright PF, Hall SL, et al.: A live attenuated bovine parainfluenza virus type 3 vaccine is safe, infectious, immunogenic and phenotypically stable in infants and children. J Infect Dis 1995, 171:1107-1114.

32. Karron RA, Makhene M, Gay K, et al.: Evaluation of a live attenuated bovine parainfluenza type 3 vaccine in two- to six-month-old infants. Pediatr Infect Dis J 1996, 15:650-654.

33. Greenberg DP, Yeh S, Yogev R, et al.: Evaluation of the safety, immunogenicity and virus shedding of an intranasal live attenuated bovine parainfluenza virus type 3 vaccine in infants. Pediatr Res 1999, 45:161A.

34. American Academy of Pediatrics: Recommended childhood immunization schedule-United States, January-December 2001. Pediatrics 2001, 107:202.

35. Zenni MK, Cheatham SH, Thompson JM, et al:: Streptococcus pneumoniae colonization in the young child: association with otitis media and resistance to penicillin. J Pediatr 1995, 127:533-537.

36. Dagan R, Muallem M, Melamed R, et al.: Reduction of pneumococcal nasopharyngeal carriage in early infancy after immunization with tetravalent pneumococcal vaccines conjugated to either tetanus toxoid or diphtheria toxoid. Pediatr Infect Dis J 1997, 16:1060-1064.

37.• Black S, Shinefield H, Fireman B, et al.: Efficacy, safety and immunogenicity of heptavalent pneumococcal conjugate vaccine in children. Pediatr Infect Dis J 2000, 19:187-195.

In this double-blind, randomized, placebo-controlled trial of 37,868 children, heptavalent PCV was $93.9 \%$ efficacious against invasive pneumococcal infections caused by vaccine serotypes. The vaccine reduced the incidence of all episodes of AOM by $6.4 \%$, visits for AOM by $7.8 \%$, and the need for ventilatory tube placement by $20.3 \%$.
$38 . \bullet$ Eskola J, Kilpi T, Palmu A, et al.: Efficacy of a pneumococcal conjugate vaccine against acute otitis media. $N$ Engl J Med 2001, 344:403-409.

In this double-blind, randomized, placebo-controlled trial of 1662 children, heptavalent PCV reduced the incidence of all causes of AOM by $6 \%$, all pneumococcal AOM by $34 \%$, and AOM caused by vaccine serotypes by $57 \%$.

39. Mbelle N, Huebner RE, Wasas AD, et al.: Immunogenicity and impact on nasopharyngeal carriage of a nonavalent pneumococcal conjugate vaccine. $J$ Infect Dis 1999, 180:1171-1176.

40. Briles DE, Hollingshead SK, Nabors GS, et al.: The potential for using protein vaccines to protect against otitis media caused by Streptococcus pneumoniae. Vaccine 2001, 19:S87-S95.

41. White P, Hermansson A, Svanborg C, et al.: Effects of active immunization with a pneumococcal surface protein (PspA) and of locally applied antibodies in experimental otitis media. ORL J Otorhinolaryngol Relat Spec 1999, 61:206-211.

42. Nabors GS, Braun PA, Herrmann DJ, et al.: Immunization of healthy adults with a single recombinant pneumococcal surface protein A (PspA) variant stimulates broadly crossreactive antibodies to heterologous PspA molecules. Vaccine 2000, 18:1743-1754

43. Foxwell RA, Kyd JM, Cripps AW: Nontypeable Haemophilus influenzae: pathogenesis and prevention. Microbiol Mol Biol Rev 1998, 62:294-308.

44. Kyd JM, Dunkley ML, Cripps AW: Enhanced respiratory clearance of nontypeable Haemophilus influenzae following mucosal immunization with P6 in a rat model. Infect Immun 1995, 63:2931-2940.

45. Yamanaka N, Faden H: Antibody response to outer membrane protein of nontypeable Haemophilus influenzae in otitisprone children. J Pediatr 1993, 122:212-218.

46. Bakaletz LO, Kennedy BJ, Novotny LA, et al.: Protection against development of otitis media induced by nontypeable Haemophilus influenzae by both active and passive immunization in a chinchilla model of virus-bacterium superinfection. Infect Immun 1999, 67:2746-2762.

47. Giebink GS: Vaccination against middle-ear bacterial and viral pathogens. Ann N Y Acad Sci 1997, 830:330-352

48. Kyd J, John A, Cripps A, Murphy TF: Investigation of mucosal immunisation in pulmonary clearance of Moraxella (Branhamella) catarrhalis. Vaccine 2000, 18:398-406.

49. McMichael JC: Vaccines for Moraxella catarrhalis. Vaccine 2001, 19:S101-S107. 